\title{
Volterra Integral Equations on Unbounded Time Scales
}

\author{
Andrejs Reinfelds ${ }^{1,2}$ and Shraddha Christian ${ }^{2}$ \\ ${ }^{1}$ Institute of Mathematics and Computer Science \\ Rīga, LV-1459, Latvia \\ ${ }^{2}$ University of Latvia, Department of Mathematics \\ Rīga, LV-1004, Latvia
}

\begin{abstract}
This paper introduces a more general result on existence, uniqueness and boundedness for solutions of nonlinear Volterra integral equation on time scales. We use Lipschitz type function, which can be an unbounded, and the Banach fixed point theorem at appropriate functional space. Furthermore it allows to get new sufficient conditions for boundedness of solutions.
\end{abstract}

AMS Subject Classifications: 45D05, 45G10, 34N05.

Keywords: Volterra integral equation on time scales, bounded solution.

\section{Introduction}

This paper introduces a more general result: existence, uniqueness, boundedness and certain growth rates for solutions of nonlinear Volterra integral equation on time scales. Kulik [8] restricted his research to the case when Lipschitz type function is constant. We generalize the results of [8,10-12] using Lipschitz type function, which can be an unbounded, and the Banach's fixed point theorem at appropriate functional space. Furthermore it allows to get new sufficient conditions for boundedness of solutions [9]. In addition, we should note articles $[1,6]$ that are very important in this direction.

The field of dynamic equations on time scales is an emerging area that has more potential created by Hilger in 1990 [5]. This new and compelling area of mathematics is more general and versatile than the traditional theories of differential and difference equations. The field of dynamic equations on time scales contains and extends the classical theory of differential, difference, integral and summation equations as special

Received March 27, 2019; Accepted September 2, 2019

Communicated by Agnieszka Malinowska 
cases. To understand the notation in this article some basic definitions are needed (for details see [2,3]).

A time scale $\mathbb{T}$ is an arbitrary non empty closed subset of the real numbers $\mathbb{R}$. Since a time scale may or may not be connected, the concept of jump operator is useful for describing the structure of the time scale under consideration and is also used in defining the delta derivative. The forward jump operator $\sigma: \mathbb{T} \rightarrow \mathbb{T}$ is defined by the equality

$$
\sigma(t)=\inf \{s \in \mathbb{T} \mid s>t\}
$$

while the backward jump operator $\rho: \mathbb{T} \rightarrow \mathbb{T}$ is defined by the equality

$$
\rho(t)=\sup \{s \in \mathbb{T} \mid s<t\} .
$$

The graininess function $\mu: \mathbb{T} \rightarrow[0,+\infty)$ is defined by

$$
\mu(t)=\sigma(t)-t
$$

The jump operators allow the classification of points in a time scale $\mathbb{T}$. If $\sigma(t)>t$, then the point $t \in \mathbb{T}$ is called right scattered while if $\rho(t)<t$, then the point $t \in \mathbb{T}$ is called left scattered. If $\sigma(t)=t$ then $t \in \mathbb{T}$ is called right dense while if $\rho(t)=t$ then $t \in \mathbb{T}$ is called left dense.

Assume $g: \mathbb{T} \rightarrow \mathbb{R}$ is a function and fix $t \in \mathbb{T}$. The delta derivative (also Hilger derivative) $g^{\Delta}(t)$ exists if for every $\varepsilon>0$ there exists a neighbourhood $U=(t-\delta, t+$ $\delta) \cap \mathbb{T}$ for some $\delta>0$ such that

$$
\left|(g(\sigma(t))-g(s))-g^{\Delta}(t)(\sigma(t)-s)\right| \leq \varepsilon|\sigma(t)-s|, \text { for all } s \in U .
$$

Take $\mathbb{T}=\mathbb{R}$ and $g$ is differentiable in the ordinary sense at $t \in \mathbb{T}$. Then $g^{\Delta}(t)=g^{\prime}(t)$ is the derivative used in standard calculus. Take $\mathbb{T}=\mathbb{Z}$. Then $g^{\Delta}(t)=\Delta g(t)$ is the forward difference operator used in difference equation.

A function $g: \mathbb{T} \rightarrow \mathbb{R}$ is called rd-continuous provided it is continuous at right dense points in $\mathbb{T}$ and its left sided limits exist at left dense points in $\mathbb{T}$.

If $F^{\Delta}(t)=g(t)$ then define the (Cauchy) delta integral by

$$
\int_{r}^{s} g(t) \Delta t=F(s)-F(r), \text { for all } r, s \in \mathbb{T} .
$$

If $\mathbb{T}=\mathbb{R}$, then

$$
\int_{r}^{s} g(t) \Delta t=\int_{r}^{s} g(t) d t
$$

while $\mathbb{T}=\mathbb{Z}$, then

$$
\int_{r}^{s} g(t) \Delta t=\sum_{t=r}^{s-1} g(t), \text { if } r, s \in \mathbb{T} \text { and } r<s .
$$


This paper considers nonlinear Volterra integral equation on time scales

$$
x(t)=f(t)+\int_{a}^{t} K(t, s, x(s)) \Delta s, \quad a, t \in I_{\mathbb{T}}=[a,+\infty) \cap \mathbb{T},
$$

where $x: I_{\mathbb{T}} \rightarrow \mathbb{R}^{n}$ is the unknown function, $f: I_{\mathbb{T}} \rightarrow \mathbb{R}^{n}$ and $K: I_{\mathbb{T}} \times I_{\mathbb{T}} \times \mathbb{R}^{n} \rightarrow \mathbb{R}^{n}$ are nonlinear functions. Equation (1.1) is known as a Volterra integral equation on time scales.

If $\mathbb{T}=\mathbb{R}$ then (1.1) becomes the familiar Volterra integral equation

$$
x(t)=f(t)+\int_{a}^{t} K(t, s, x(s)) d s, \quad t \in[a,+\infty) .
$$

If $\mathbb{T}=\mathbb{Z}$ then (1.1) becomes the well-known Volterra summation equation

$$
x(t)=f(t)+\sum_{s=a}^{t-1} K(t, s, x(s)), \quad t \in\{a, a+1, \cdots\} .
$$

\section{Existence and Uniqueness of Solutions on Unbounded Time Scales}

Consider the integral equation (1.1). We now construct the appropriate Banach space for our analysis. Let $\beta: I_{\mathbb{T}} \rightarrow \mathbb{R}$ be a regressive and rd-continuous scalar function. The Cauchy initial value problem for scalar linear equation

$$
x^{\Delta}=\beta(t) x, \quad x(a)=1
$$

has the unique solution $e_{\beta}(\cdot, a): I_{\mathbb{T}} \rightarrow \mathbb{R}[2]$. More explicitly, using the cylinder transformation the exponential function $e_{\beta}(\cdot, a)$ is given by

$$
e_{\beta}(t, a)=\exp \left(\int_{a}^{t} \xi_{\mu(s)}(\beta(s)) \Delta s\right)
$$

where

$$
\xi_{h}(z)= \begin{cases}z, & h=0 \\ \frac{1}{h} \log (1+h z), & h>0 .\end{cases}
$$

Karpuz [7] gave the alternative definition for the time scales exponential function.

Observe that we have important estimate [4]

$$
1 \leq 1+\int_{a}^{t} \beta(s) \Delta s \leq e_{\beta}(t, a) \leq \exp \left(\int_{a}^{t} \beta(s) \Delta s\right)
$$

for all $t \in I_{\mathbb{T}}$. 
Let $|\cdot|$ denote the Euclidean norm on $\mathbb{R}^{n}$. We will consider the linear space of continuous functions $C\left(I_{\mathbb{T}} ; \mathbb{R}^{n}\right)$ such that

$$
\sup _{t \in I_{\mathbb{T}}} \frac{|x(t)|}{e_{\beta}(t, a)}<\infty
$$

and denote this special space by $C_{\beta}\left(I_{\mathbb{T}} ; \mathbb{R}^{n}\right)$. The space $C_{\beta}\left(I_{\mathbb{T}} ; \mathbb{R}^{n}\right)$ can be endowed with a suitable norm, expressly

$$
\|x\|_{\beta}=\sup _{t \in I_{\mathbb{T}}} \frac{|x(t)|}{e_{\beta}(t, a)} .
$$

We generalize and at the same time simplify the results $[8,10-12]$ assuming $L$ can be an unbounded rd-continuous function.

Theorem 2.1. Consider the integral equation (1.1). Let $K: I_{\mathbb{T}} \times I_{\mathbb{T}} \times \mathbb{R}^{n} \rightarrow \mathbb{R}^{n}$ be jointly continuous in its first and third variables and rd-continuous in its second variable, $f: I_{\mathbb{T}} \rightarrow \mathbb{R}^{n}$ be continuous, $L: I_{\mathbb{T}} \rightarrow \mathbb{R}$ be rd-continuous, $\gamma>1$ and $\beta(s)=$ $L(s) \gamma$. If

$$
\begin{gathered}
|K(t, s, p)-K(t, s, q)| \leq L(s)|p-q|, \quad p, q \in \mathbb{R}^{n}, \quad s<t, \\
m=\sup _{t \in I_{\mathbb{T}}} \frac{1}{e_{\beta}(t, a)}\left|f(t)+\int_{a}^{t} K(t, s, 0) \Delta s\right|<\infty,
\end{gathered}
$$

then the integral equation (1.1) has a unique solution $x \in C_{\beta}\left(I_{\mathbb{T}} ; \mathbb{R}^{n}\right)$.

Proof. Consider the following equivalent formulation of (1.1), namely

$$
x(t)=\left(f(t)+\int_{a}^{t} K(t, s, 0) \Delta s\right)+\int_{a}^{t}(K(t, s, x(s))-K(t, s, 0)) \Delta s .
$$

We will show that (2.4) has a unique solution and thus (1.1) must also have a unique solution.

Let $L: I_{\mathbb{T}} \rightarrow \mathbb{R}$ be the function defined in (2.2) and let $\beta(s)=L(s) \gamma$, where $\gamma>1$. Consider the Banach space $C_{\beta}\left(I_{\mathbb{T}} ; \mathbb{R}^{n}\right)$ and let the operator $F$ be defined by

$$
[F x](t)=\left(f(t)+\int_{a}^{t} K(t, s, 0) \Delta s\right)+\int_{a}^{t}(K(t, s, x(s))-K(t, s, 0)) \Delta s .
$$

Fixed point of $F$ will be solution to (2.4). Thus we want to prove that there exists a unique $x$ such that $F x=x$. We show that $F: C_{\beta}\left(I_{\mathbb{T}} ; \mathbb{R}^{n}\right) \rightarrow C_{\beta}\left(I_{\mathbb{T}} ; \mathbb{R}^{n}\right)$. Let $x \in C_{\beta}\left(I_{\mathbb{T}} ; \mathbb{R}^{n}\right)$. Taking norms in (2.4), we obtain

$$
\|F x\|_{\beta}=\sup _{t \in I_{\mathbb{T}}} \frac{1}{e_{\beta}(t, a)}\left|f(t)+\int_{a}^{t} K(t, s, 0) \Delta s+\int_{a}^{t}(K(t, s, x(s))-K(t, s, 0)) \Delta s\right|
$$




$$
\begin{aligned}
& \leq m+\sup _{t \in I_{\mathbb{T}}} \frac{1}{e_{\beta}(t, a)} \int_{a}^{t} L(s)|x(s)| \Delta s \\
& =m+\sup _{t \in I_{\mathbb{T}}} \frac{1}{e_{\beta}(t, a)} \int_{a}^{t} L(s) e_{\beta}(s, a) \frac{|x(s)|}{e_{\beta}(s, a)} \Delta s \\
& \leq m+\|x\|_{\beta} \sup _{t \in I_{\mathbb{T}}} \frac{1}{e_{\beta}(t, a)} \int_{a}^{t} L(s) e_{\beta}(s, a) \Delta s \\
& =m+\frac{\|x\|_{\beta}}{\gamma} \sup _{t \in I_{\mathbb{T}}} \frac{1}{e_{\beta}(t, a)} \int_{a}^{t} \gamma L(s) e_{\beta}(s, a) \Delta s \\
& =m+\frac{\|x\|_{\beta}}{\gamma} \sup _{t \in I_{\mathbb{T}}} \frac{1}{e_{\beta}(t, a)} \int_{a}^{t} e_{\beta}^{\Delta}(s, a) \Delta s \\
& =m+\frac{\|x\|_{\beta}}{\gamma} \sup _{t \in I_{\mathbb{T}}} \frac{1}{e_{\beta}(t, a)}\left[e_{\beta}(t, a)-1\right] \\
& =m+\frac{\|x\|_{\beta}}{\gamma} \sup _{t \in I_{\mathbb{T}}}\left(1-\frac{1}{e_{\beta}(t, a)}\right)=m+\frac{\|x\|_{\beta}}{\gamma}<\infty .
\end{aligned}
$$

Hence we see that $F: C_{\beta}\left(I_{\mathbb{T}} ; \mathbb{R}^{n}\right) \rightarrow C_{\beta}\left(I_{\mathbb{T}} ; \mathbb{R}^{n}\right)$. We show that $F$ is a contraction map with contraction constant $\alpha=1 / \gamma<1$ and then Banach's fixed point theorem will apply. For any $u, v \in C_{\beta}\left(I_{\mathbb{T}} ; \mathbb{R}^{n}\right)$

$$
\begin{aligned}
\|F u-F v\|_{\beta} & =\sup _{t \in I_{\mathbb{T}}} \frac{|[F u](t)-[F v](t)|}{e_{\beta}(t, a)} \\
& \leq \sup _{t \in I_{\mathbb{T}}} \frac{1}{e_{\beta}(t, a)} \int_{a}^{t}|K(t, s, u(s))-K(t, s, v(s))| \Delta s \\
& \leq \sup _{t \in I_{\mathbb{T}}} \frac{1}{e_{\beta}(t, a)} \int_{a}^{t} L(s)|u(s)-v(s)| \Delta s \\
& =\sup _{t \in I_{\mathbb{T}}} \frac{1}{e_{\beta}(t, a)} \int_{a}^{t} L(s) e_{\beta}(s, a) \frac{|u(s)-v(s)|}{e_{\beta}(s, a)} \Delta s \\
& \leq\|u-v\|_{\beta} \sup _{t \in I_{\mathbb{T}}} \frac{1}{e_{\beta}(t, a)} \int_{a}^{t} L(s) e_{\beta}(s, a) \Delta s \\
& =\frac{\|u-v\|_{\beta}}{\gamma} \sup _{t \in I_{\mathbb{T}}} \frac{1}{e_{\beta}(t, a)} \int_{a}^{t} \gamma L(s) e_{\beta}(s, a) \Delta s \\
& =\frac{\|u-v\|_{\beta}}{\gamma} \sup _{t \in I_{\mathbb{T}}} \frac{1}{e_{\beta}(t, a)} \int_{a}^{t} e_{\beta}^{\Delta}(s, a) \Delta s \\
& =\frac{\|u-v\|_{\beta}}{\gamma} \sup _{t \in I_{\mathbb{T}}}\left[1-\frac{1}{e_{\beta}(t, a)}\right] \\
& \leq \frac{\|u-v\|_{\beta}}{\gamma}=\alpha\|u-v\|_{\beta} .
\end{aligned}
$$


As $\alpha<1$, we see that $F$ is a contraction map and so Banach's fixed point theorem applies, yielding the existence of a unique fixed point $x$ of $F$.

Example 2.2. Consider the scalar integral equation

$$
x(t)=t^{2}+\int_{a}^{t}(s+\sigma(s))\left[x(s)^{2}+1\right]^{\frac{1}{2}} \Delta s, \quad a, t \in I_{\mathbb{T}}=[a,+\infty) \cap \mathbb{T}, a \geq 0 .
$$

We claim that this integral equation has a unique solution for arbitrary $\mathbb{T}$.

Proof. We will use the above Theorem 2.1 and make use of the fact that $K(t, s, p)=$ $(s+\sigma(s))\left(p^{2}+1\right)^{\frac{1}{2}}$ has a bounded partial derivative with respect to $p$ everywhere consider

$$
\begin{aligned}
|K(t, s, p)-K(t, s, q)| & =(s+\sigma(s))\left|\left(p^{2}+1\right)^{\frac{1}{2}}-\left(q^{2}+1\right)^{\frac{1}{2}}\right| \\
& \leq(s+\sigma(s)) \sup _{r \in \mathbb{R}}\left|\frac{r}{\left(r^{2}+1\right)^{\frac{1}{2}}}\right||p-q| \\
& \leq(s+\sigma(s))|p-q| .
\end{aligned}
$$

Here we used the mean value theorem. So (2.2) holds with $L(s)=s+\sigma(s)$. For a choice of, say, $\gamma=2$ we then have $\beta(s)=2(s+\sigma(s))$ and considering that

$$
\int_{a}^{t}(s+\sigma(s)) \Delta s=t^{2}-a^{2}
$$

and $e_{\beta}(t, a) \geq 1+t^{2}-a^{2}$ it is not difficult to verify (2.3) holds. The result now follows from the Theorem 2.1.

\section{Bounded Solutions}

We now present results concerning boundedness of solutions of Volterra integral equations.

Theorem 3.1. Consider the Volterra integral equation (1.1) satisfying conditions of Theorem 2.1. If in addition

$$
\sup _{t \in I_{\mathbb{T}}} \int_{a}^{t} L(s) \Delta s=\nu<\infty
$$

and

$$
m_{1}=\sup _{t \in I_{\mathbb{T}}}\left|f(t)+\int_{a}^{t} K(t, s, 0) \Delta s\right|<\infty
$$

then the unique solution of Volterra integral equation (1.1) on time scales that are unbounded above is bounded

$$
\sup _{t \in I_{\mathbb{T}}}|x(t)|<+\infty
$$


Proof. In our case $\beta(s)=\gamma L(s)$. Using the estimate (2.1) and condition (3.1) we get that

$$
1 \leq e_{\beta}(t, a) \leq \exp \left(\int_{a}^{t} \beta(s) \Delta s\right) \leq \exp (\gamma \nu)
$$

So norm $\|x\|_{\beta}$ and supremum norm $\sup _{t \in I_{\mathbb{T}}}|x(t)|$ are equivalent at space $C_{\beta}\left(I_{\mathbb{T}} ; \mathbb{R}^{n}\right)$. This means that Volterra integral equation solution is bounded if additional conditions (3.1) and (3.2) fulfilled and $\nu>0$.

Example 3.2. Consider the scalar integral equation

$$
x(t)=2+\int_{a}^{t} \frac{\left(x(s)^{2}+1\right)^{\frac{1}{2}}}{s \sigma(s)} \Delta s, \quad a, t \in I_{\mathbb{T}}=[a,+\infty) \cap \mathbb{T}, a>0 .
$$

We claim that this integral equation has a bounded solution for arbitrary $\mathbb{T}$.

Proof. Let us note that

$$
\int_{a}^{t} \frac{\Delta s}{s \sigma(s)}=a^{-1}-t^{-1}<a^{-1}
$$

The result now follows from the Theorem 3.1.

Let us consider Volterra integral equation (1.1) with more general Lipschitz conditions. This allows us to supplemented conditions of existence of bounded solution [9].

Theorem 3.3. Consider the integral equation (1.1) satisfying conditions of Theorem 2.1 and condition (3.2). Let $L_{1}: I_{\mathbb{T}} \times I_{\mathbb{T}} \rightarrow \mathbb{R}$ be continuous in its first and rd-continuous in its second variable such that

$$
|K(t, s, p)-K(t, s, q)| \leq L_{1}(t, s)|p-q|, \quad p, q \in \mathbb{R}^{n}, \quad s<t,
$$

and assume that there exists $t_{1} \in I_{\mathbb{T}}$ such that

$$
\begin{gathered}
m_{2}=\sup _{t \in I_{\mathbb{T}}} \int_{a}^{t_{1}} L_{1}(t, s) \Delta s<\infty, \\
\sup _{t \in\left[t_{1},+\infty\right) \cap \mathbb{T}} \int_{t_{1}}^{t} L_{1}(t, s) \Delta s \leq \lambda<1 .
\end{gathered}
$$

Then the integral equation (1.1) has a unique bounded solution.

Proof. According to Theorem 2.1, Volterra integral equation (1.1) has a solution on time scales that are unbounded above. Consider the following equivalent formulation of $(1.1)$

$$
x(t)=\left(f(t)+\int_{a}^{t} K(t, s, 0) \Delta s\right)+\int_{a}^{t}(K(t, s, x(s))-K(t, s, 0)) \Delta s .
$$


Let

$$
M=m_{1}+m_{2} \sup _{s \in\left[a, t_{1}\right] \cap \mathbb{T}}|x(s)|
$$

and suppose that

$$
\sup _{t \in I_{\mathbb{T}}}|x(t)| \leq \frac{M}{1-\lambda}
$$

Then

$$
\begin{aligned}
|F[x(t)]| & \leq \sup _{t \in I_{\mathbb{T}}}\left|f(t)+\int_{a}^{t} K(t, s, 0) \Delta s\right| \\
& +\int_{a}^{t_{1}}|K(t, s, x(s))-K(t, s, 0)| \Delta s \\
& +\int_{t_{1}}^{t}|K(t, s, x(s))-K(t, s, 0)| \Delta s \\
& \leq m_{1}+\int_{a}^{t_{1}} L_{1}(t, s)|x(s)| \Delta s+\int_{t_{1}}^{t} L_{1}(t, s)|x(s)| \Delta s \\
& \leq M+\int_{t_{1}}^{t} L_{1}(t, s)|x(s)| \Delta s \leq M+\lambda \frac{M}{1-\lambda}=\frac{M}{1-\lambda} .
\end{aligned}
$$

So we have that

$$
\sup _{t \in I_{\mathbb{T}}}|F[x(t)]| \leq \frac{M}{1-\lambda} .
$$

It follows that for unique fixed point of operator $F$

$$
\sup _{t \in I_{\mathbb{T}}}|x(t)| \leq \frac{M}{1-\lambda} .
$$

This completes the proof.

\section{References}

[1] M. Adivar and Y.N. Raffoul, Existence of resolvent for Volterra integral equations on time scales, Bull. Aust. Math. Soc. 82 (2010), no. 1, 139-155.

[2] M. Bohner and A. Peterson. Dynamic Equations on Time Scales. An Introduction with Applications. Birkhäuser, Boston/Basel/Berlin, 2001.

[3] M. Bohner and A. Peterson. Advances in Dynamic Equations on Time Scales. Birkhäuser, Boston/Basel/Berlin, 2003.

[4] M. Bohner, Some oscillation criteria for first order delay dynamic equations, Far East Journal of Applied Mathematics 18 (2005), no. 3, 289-304. 
[5] S. Hilger, Analysis on measure chains. A unified approach to continuous and discrete calculus, Results Math. 18 (1990)), no. 1-2, 18-56.

[6] B. Karpuz, Volterra theory on time scales, Results Math. 65 (2014), no. 3-4, 263292.

[7] B. Karpuz, Analyticity of the complex time scale exponential, Complex Anal. Oper. Theory 11 (2017), no. 1, 21-34.

[8] T. Kulik and C.C. Tisdell, Volterra integral equations on time scales. Basic qualitative and quantitative results with applications to initial value problems on unbounded domains, Int. J. Difference Equ. 3 (2008), no. 1, 103-133.

[9] E. Messina and A. Vecchio, Stability and convergence of solutions to Volterra integral equation on time scales, Discrete Dynamics in Nature and Society, (2015), Article ID 612156.

[10] D.B. Pachpatte, On a nonstandard Volterra type dynamic integral equation on time scales, Electron. J. Qual. Theory Differ. Equ. 72 (2009).

[11] D.B. Pachpatte, Properties of some dynamic integral equation on time scales, Ann. Funct. Anal. 4 (2013), no. 2, 12-26.

[12] I.L.D. dos Santos, On Volterra integral equations on time scales, Mediter. J. Math. 12 (2015), no. 2, 471-480. 
